\title{
On the irreducibility of certain polynomials with coefficients as products of terms in an arithmetic progression
}

\author{
by
}

Carrie E. Finch (Lexington, VA) and N. Saradha (Mumbai)

1. Introduction. In 1929, Schur [10] used prime ideals in algebraic number fields to prove that the Taylor polynomials for the exponential function, with some possible variations in the coefficients, are irreducible.

TheOrem 1. Let $m$ be a positive integer and let $a_{0}, \ldots, a_{m}$ be arbitrary integers with $\left|a_{0}\right|=\left|a_{m}\right|=1$. Then

$$
a_{m} \frac{x^{m}}{m !}+a_{m-1} \frac{x^{m-1}}{(m-1) !}+\cdots+a_{1} x+a_{0}
$$

is irreducible over the rationals.

Filaseta [7] used Newton polygons to obtain Schur's result, and also strengthened the result by allowing more possible values for the leading coefficient than just those of absolute value 1 .

TheOREM 2. Let $m$ be a positive integer and let $a_{0}, \ldots, a_{m}$ be arbitrary integers with $\left|a_{0}\right|=1$ and $0<\left|a_{m}\right|<m$. Then the polynomial given in (1.1) is irreducible over the rationals except when

$$
a_{m}= \pm 5 \text { and } m=6 \text { or } a_{m}= \pm 7 \text { and } m=10 .
$$

Filaseta's use of Newton polygons to demonstrate the irreducibility of the polynomials in (1.1) is based on the theorem of Dumas [3] regarding the construction of the Newton polygon of the product of two polynomials. In particular, Filaseta employs the following useful lemma, from [6].

Lemma 1. Let $k$ and $l$ be integers with $k>l \geq 0$. Suppose $g(x)=$ $\sum_{j=0}^{n} b_{j} x^{j} \in \mathbb{Z}[x]$ and $p$ is a prime such that $p \nmid b_{n}, p \mid b_{j}$ for all $j \in$ $\{0,1, \ldots, n-l-1\}$, and the rightmost edge of the Newton polygon for $g(x)$

2010 Mathematics Subject Classification: Primary 12E05; Secondary 12D05.

Key words and phrases: arithmetic progressions, greatest prime factor, irreducibility of polynomials, Newton polygons. 
with respect to $p$ has slope $<1 / k$. Then for any integers $a_{0}, a_{1}, \ldots, a_{n}$ with $\left|a_{0}\right|=\left|a_{n}\right|=1$, the polynomial $f(x)=\sum_{j=0}^{n} a_{j} b_{j} x^{j}$ cannot have a factor with degree in the interval $[l+1, k]$.

From this lemma, we see that using Newton polygons to eliminate the possibility of factors of a particular degree hinges on finding primes that divide certain coefficients of the polynomial. To obtain Theorem 2, Filaseta appeals to a result of Ecklund, Eggleton, Erdös and Selfridge [5] on prime divisors of binomial coefficients. We refer to [7] for details. Moreover, using the same tools, Allen and Filaseta [1, 2] proved the following result.

TheOREM 3. Let $m>1$ and $a_{0}, \ldots, a_{m}$ denote arbitrary integers with $\left|a_{0}\right|=1$.

(i) Suppose $m+1=k^{\prime} 2^{u}$ with $k^{\prime}$ odd and $(m+1) m=k^{\prime \prime} 2^{v} 3^{w}$ with $\operatorname{gcd}\left(k^{\prime \prime}, 6\right)=1$. Let $0<\left|a_{m}\right|<\min \left\{k^{\prime}, k^{\prime \prime}\right\}$. Then

$$
a_{m} \frac{x^{m}}{(m+1) !}+a_{m-1} \frac{x^{m-1}}{m !}+\cdots+a_{2} \frac{x^{2}}{2 !}+a_{1} x+a_{0}
$$

is irreducible over the rationals.

(ii) Suppose $0<\left|a_{m}\right|<2 m-1$. Then

$$
\begin{aligned}
a_{m} \frac{x^{2 m}}{1 \cdot 3 \cdots(2 m-1)}+a_{m-1} \frac{x^{2 m-2}}{1 \cdot 3 \cdots(2 m-3)} & +\cdots \\
& +a_{2} \frac{x^{4}}{1 \cdot 3}+a_{1} \frac{x^{2}}{1}+a_{0}
\end{aligned}
$$

is irreducible over the rationals.

We observe that the common thread among the polynomials in (1.1), $(1.2)$, and $(1.3)$ is that the denominators of the coefficients are products of integers in an arithmetic progression; in the case of $(1.1)$ and $(1.2)$, we see an arithmetic progression with initial term 1 and common difference 1 , and in 1.3 we see an arithmetic progression with initial term 1 and common difference 2 . In this paper, we prove analogous results by considering denominators which are again products of integers in an arithmetic progression with initial term an odd integer $a$ and common difference 2. Let

$$
\begin{aligned}
& f(x)=\frac{x^{m}}{a(a+2) \cdots(a+2(m-1))}+\cdots+\frac{x^{2}}{a(a+2)}+\frac{x}{a}+1, \\
& g(x)=a_{m} \frac{x^{m}}{a(a+2) \cdots(a+2(m-1))} \\
& +a_{m-1} \frac{x^{m-1}}{a(a+2) \cdots(a+2(m-2))}+\cdots+a_{2} \frac{x^{2}}{a(a+2)}+a_{1} \frac{x}{a}+a_{0} .
\end{aligned}
$$

Letting $P(n)$ denote the greatest prime factor of the positive integer $n$ (putting $P(1)=1$ ), we prove the following results. 
Theorem 4. Let $a \geq 1$ be an odd integer, $\max \{a, 110\} \leq k \leq m / 2$ and $a_{0}, \ldots, a_{m}$ be arbitrary integers with $P\left(a_{0} a_{m}\right) \leq 2 k+a$. Then $f(x)$ and $g(x)$ do not have a factor of degree $k$.

Now we restrict to $1 \leq a<29$. We have

Theorem 5. Let $a$ be an odd integer with $1 \leq a<29$, and let $m>1$ and $a_{0}, \ldots, a_{m}$ be arbitrary integers with $P\left(a_{0} a_{m}\right) \leq a+4$. Then

(i) $f(x)$ has no factor of degree $\geq 2$,

(ii) $g(x)$ has no factor of degree $\geq 3$, and

(iii) $g(x)$ has no factor of degree 2 except perhaps when

$$
(a, m) \in\{(21,4),(19,59),(5,121),(19,114),(21,113),(21,163),(21,554)\} .
$$

Further if there exists a prime $p \geq a+2$ dividing $a+2(m-1)$, then $f(x)$ has no linear factor. Also if such a prime $p$ does not divide $a_{0} a_{m}$, then $g(x)$ has no linear factor.

When $(a, m)=(21,4)$, by choosing $a_{0}=a_{4}=1, a_{1}=-15, a_{2}=-140$, $a_{3}=35$, we see that

$$
\begin{aligned}
g(x) & =\frac{x^{4}}{21 \cdot 23 \cdot 25 \cdot 27}+\frac{35 x^{3}}{21 \cdot 23 \cdot 25}-\frac{140 x^{2}}{21 \cdot 23}-\frac{15 x}{21}+1 \\
& =\frac{1}{326025}\left(x^{2}-90 x-315\right)\left(x^{2}+1035 x-1035\right) .
\end{aligned}
$$

We thank the referee for providing this example. As in the proofs of Theorems 2 and 3, our method also depends on the use of Dumas' theorem on Newton polygons for the irreducibility of polynomials. On the other hand, we do not use results from Ecklund, Eggleton, Erdös, and Selfridge [5]. Instead, we establish a Sylvester type result on the greatest prime factor of a product of several consecutive terms in an arithmetic progression. This result is of interest independent of its application to establish Theorems 4 and 5 . We show for instance that for any $k \geq 2$,

$$
P(n(n+2) \cdots(n+2(k-1)))>2 k+a
$$

if $n \geq 2 k+a$, where $n$ is odd and $a$ is a positive odd integer less than 29, except for an explicitly given set of values of $(n, k, a)$. This result depends on a result of Lehmer [8] and several computations. The above assertion is also true for any odd $a$ provided $k$ is large; see Lemma 5 . As an application of Theorem 5 we give another criterion for the irreducibility of $(1.3)$.

Corollary 6. Let $P\left(a_{0} a_{m}\right) \leq 5$. Suppose there exists a prime $p$ such that

$$
p \mid(2 m-1) \quad \text { and } \quad p \nmid a_{0} a_{m} .
$$

Then the polynomial given in (1.3) is irreducible over the rationals. 
Let $a$ be an even integer equal to $2 b$, say. Then $f(x)$ and $g(x)$ can be transformed into a polynomial of the form

$$
a_{m}^{\prime} \frac{x^{m}}{b(b+1) \cdots(b+m-1)}+\cdots+a_{1}^{\prime} \frac{x}{b}+a_{0}^{\prime}
$$

with $a_{m}^{\prime}=1, a_{0}^{\prime}, \ldots, a_{m-1}^{\prime}$ integers. The case $b=1$ and $\left|a_{0}^{\prime}\right|=1$ is Schur's polynomial given in (1.1). For some results on the factors of such polynomials, we refer to [11. We will not deal with this case in the present paper.

The remainder of this paper is organized as follows. In Section 2, we prove the result about the greatest prime factor of a product of consecutive terms in arithmetic progression. In Section 3, we use Newton polygons to exclude some factors of the polynomials in question. In particular cases, all factors of degree $\geq 2$ are excluded. Theorem 4 comes out as a consequence of Lemmas 5 and 11. In Section 4 we discuss linear factors and prove Theorem 5 from Theorems 8 and 9 .

2. Greatest prime factor of a product of integers in arithmetic progression. The letters $n, d, k$ denote positive integers with $\operatorname{gcd}(n, d)=1$. Set $\Delta=n(n+d) \cdots(n+(k-1) d)$. Let $\pi(n)$ denote the number of primes $\leq n$ and $\pi_{d}(n)$ be the number of primes $\leq n$ that are co-prime to $d$. Let $\nu_{p}(n)$ denote the power of the prime $p$ in $n$ and $p_{i}$ denote the $i$ th prime. In this section, we obtain lower bounds on $P(\Delta)$, and conclude the section with Theorem 8, a particular result for $P(\Delta)$ when $d=2$.

We state without proof our first lemma. See [9] for details.

Lemma 2. For $0 \leq i<k$, suppose $P(n+i d) \leq c_{0} k$. Let $S=$ $\{n, n+d, \ldots, n+(k-1) d\}$. For every prime $p \leq c_{0} k$ with $p \nmid d$, choose $n+i_{p} d \in S$ such that $p$ does not appear to a higher power in the factorization of any other element of $S$. Let $S_{1}$ be the subset of $S$ obtained by deleting from $S$ all $n+i_{p} d$ with $p \leq c_{0} k$ and $p \nmid d$. Then

$$
\prod_{n+i d \in S_{1}}(n+i d) \leq(k-1) ! \prod_{p \mid d} p^{-\nu_{p}((k-1) !)} .
$$

When $d=1$, the product on the right hand side is taken as 1 . In the next lemma, inequality (i) is an easy consequence of the formula of Legendre on the $\nu_{p}((k-1) !)$. The estimate for $\pi(x)$ in (ii) is due to Dusart [4].

LEMMA 3.

(i) $\nu_{p}((k-1) !) \geq \frac{k-p}{p-1}-\frac{\log (k-1)}{\log p}$.

(ii) $\pi(x) \leq \frac{x}{\log x}\left(1+\frac{1.2762}{\log x}\right)$ for $x>1$. 
Lemma 4. Let $k \geq 2, c_{0}>1, c_{1}>0, d \geq 1$ and $k-\pi_{d}\left(c_{0} k\right) \geq 1$. Suppose $n \geq c_{1} k d$ and $P(\Delta) \leq c_{0} k$. Then

$$
\left(c_{1} d\right)^{k-\pi_{d}\left(c_{0} k\right)} \leq k^{\pi_{d}\left(c_{0} k\right)} \prod_{p \mid d} p^{-\nu_{p}((k-1) !)} .
$$

Proof. Observe that $\Delta$ is not divisible by primes dividing $d$ and that every prime $>k$ may divide only one term of $\Delta$. Hence there are at least $k-\pi_{d}\left(c_{0} k\right)+\pi_{d}(k)$ terms which are divisible only by primes $\leq k$. By deleting a term in which a prime $p \leq k, p \nmid d$ appears to the maximum power, using the notation from Lemma 2, we see that $\left|S_{1}\right| \geq k-\pi_{d}\left(c_{0} k\right) \geq 1$. We set $t:=\left|S_{1}\right|-1$. We arrange the elements of $S_{1}$ as

$$
n+i_{0} d<n+i_{1} d<\cdots<n+i_{t} d .
$$

Then by Lemma 2 ,

$$
\prod_{v=0}^{t}\left(n+i_{v} d\right) \leq(k-1) ! \prod_{p \mid d} p^{-\nu_{p}((k-1) !)} .
$$

This gives

$$
n^{k-\pi_{d}\left(c_{0} k\right)} \leq n(n+d) \cdots\left(n+\left(k-\pi_{d}\left(c_{0} k\right)-1\right) d\right) \leq(k-1) ! \prod_{p \mid d} p^{-\nu_{p}((k-1) !)} .
$$

Since $n \geq c_{1} k d$, we get

$$
\left(c_{1} d\right)^{k-\pi_{d}\left(c_{0} k\right)} k^{k-\pi_{d}\left(c_{0} k\right)} \leq k^{k} \prod_{p \mid d} p^{-\nu_{p}((k-1) !)},
$$

which gives the assertion of the lemma.

Putting together the inequalities from Lemma 3 with the result in Lemma 4 and observing that $\pi_{d}\left(c_{0} k\right) \leq \pi\left(c_{0} k\right)$, we obtain the following result.

Corollary 7 . Let $k \geq 2, c_{0}>1, c_{1}>0, d=p$ prime and $k-\pi_{p}\left(c_{0} k\right) \geq 1$. Suppose that $n \geq c_{1} k p$ and $P(\Delta) \leq c_{0} k$. Let

$$
f(k, p)= \begin{cases}0 & \text { if } p \geq k, \\ \frac{1}{p-1}-\frac{p}{k(p-1)}-\frac{\log (k-1)}{k \log p} & \text { otherwise. }\end{cases}
$$

Then

$$
p \leq \exp \left[\frac{c_{0}+\frac{1.2762 c_{0}}{\log c_{0} k}-\left(1-\frac{c_{0}}{\log c_{0} k}-\frac{1.2762 c_{0}}{\left(\log c_{0} k\right)^{2}}\right) \log c_{1}}{1-\frac{c_{0}}{\log c_{0} k}-\frac{1.2762 c_{0}}{\left(\log c_{0} k\right)^{2}}+f(k, p)}\right] .
$$


For the rest of this section, we restrict our attention to arithmetic progressions with common difference $d=2$. We denote

$$
\Delta_{2}=n(n+2) \cdots(n+2(k-1)) \quad \text { with } n \text { odd } .
$$

Before we state the next lemma, we note that Allen and Filaseta [1] showed that for every $n \geq 213$, there exists a prime $p \in(n, 1.05 n]$. We will use this result in the next lemma.

Lemma 5. Let $a \geq 1$ be an odd integer, $k \geq \max \{a, 110\}$ and $n \geq 2 k+a$. Then $P\left(\Delta_{2}\right)>2 k+a$.

Proof. Suppose $P\left(\Delta_{2}\right) \leq 2 k+a$. First assume that $n \geq 40(k-1)$. Note that $2+a / k \leq 3$ since $a \leq k$. We apply Corollary 7 with $c_{0}=3$ and $c_{1}=19.5$. Note that $\pi\left(c_{0} k\right)<k$ since $k \geq 110$. We find that the right hand side of the inequality in Corollary 7 is a decreasing function of $k$ since each term involving $k$ is a decreasing function of $k$. Hence if the inequality is not valid for some $k=k_{0}$, then it is not valid for any $k>k_{0}$. We check that the inequality is not valid for $k_{0}=110$. This proves the assertion of the lemma for $n \geq 40(k-1)$.

Next we assume that $n<40(k-1)$. Note that $n \geq 213$. Then there exists a prime $p$ in $\{n+2, \ldots, n+2(k-1)\}$ since the interval $(n, 1.05 n]$ is contained in $(n, n+2(k-1)]$ as $n<40(k-1)$. Further this prime exceeds $n \geq 2 k+a$, by assumption. Thus $P\left(\Delta_{2}\right)>2 k+a$.

Now we restrict to odd $a<29$.

Lemma 6. Let $1 \leq a<29$, a odd, $k \geq 31$ and $n \geq 2 k+a$. Then $P\left(\Delta_{2}\right)>2 k+29$.

Proof. Suppose $P\left(\Delta_{2}\right) \leq 2 k+29$. We follow the argument as in Lemma 5 . First let $n \geq 40(k-1)$. We apply Corollary 7 with $c_{0}=2+29 / k$ and $c_{1}=19.5$. We check that the inequality in Corollary 7 is not valid for $k_{0}=100$. Thus we may assume that $k \leq 99$. Now we check that the inequality (2.1) with actual values of the $\pi$-function is invalid for all $31 \leq k \leq 99$.

Next we assume that $213 \leq n<40(k-1)$. Since now $(n, 1.05 n] \subset$ $(n, n+2(k-1)]$, there exists a prime $\geq 2 k+a$ dividing $\Delta_{2}$. Hence we may assume that $n<213$. Then we need only consider $2 k+a \leq n<213$ with $n$ odd. For these finitely many values of $n$ and $k$, we check directly that the assertion of the lemma is true.

Let $T$ be the set of all integers $M \geq 1$ with $P(M(M+2)) \leq 31$. Table 1 below shows 101 such integers put in groups according to the largest prime factor of $M(M+2)$. It follows from Lehmer's work [8] that if $n>1$ is an 
integer, then $P(n(n+2)) \geq 37$ except when $n=M$ with $M$ given by Table 1 . Thus Table 1 gives all the integers of $T$.

\section{Table 1}

\begin{tabular}{rl}
\hline \multicolumn{1}{c}{$p$} & Integers $M$ with $P(M(M+2))=p$ \\
\hline 3 & 1 \\
5 & 3,25 \\
7 & $5,7,243$ \\
11 & $9,33,75$ \\
13 & $11,13,63,273,845,1573$ \\
17 & $15,49,117,119,187,1375$ \\
19 & $17,19,55,133,169,245,323,361,625,663,1615,3211,3969$ \\
23 & $21,23,115,207,253,297,343,1125,1309,2185,2275,2873$, \\
& $3703,6875,8073,9315,18513,41743,57475,1128125,1447873$ \\
29 & $27,85,143,145,375,435,493,665,2871,8379,9945,12673$, \\
& $14875,16443,24563,41325,45617,87723,184875$ \\
31 & $29,31,91,93,153,341,403,525,527,713,897,1083,1519$, \\
& $1953,2695,3625,4123,5423,7161,19435,22475,86273$, \\
& $130975,203203,2509045,3322053,287080365$ \\
\hline
\end{tabular}

The next three lemmas deal with the complementary case of Lemma 6 when $k \leq 30$.

Lemma 7. Let $2 \leq k \leq 30$. Suppose no $M \in T$ is of the form $n+4 j$ for any $j$ with $0 \leq j \leq(k-2) / 2$. Then $P\left(\Delta_{2}\right) \geq 2 k+29$.

Proof. We divide the integers $n, n+2, \ldots, n+2(k-1)$ into pairs

$$
(n, n+2),(n+4, n+6), \ldots
$$

Note that there are at least $[k / 2]$ pairs. By hypothesis, none of these pairs coincides with $(M, M+2)$ for any $M \in T$. Then the product of integers in each pair in $(2.2)$ has a prime factor $\geq 37$. Since these integers are in a block of length at most 30 , we see that each pair in 2.2 must have a distinct prime $\geq 37$ dividing their product. Thus $\Delta_{2}$ is divisible by at least $[k / 2]$ primes $\geq 37$. Hence

$$
P\left(\Delta_{2}\right) \geq p_{[k / 2]+11} .
$$

We check that $p_{[k / 2]+11} \geq 2 k+29$ for $2 \leq k \leq 30$, which completes the proof of the lemma.

Lemma 8. Let $3 \leq k \leq 30$ and $n \geq 2 k+29$. Then $P\left(\Delta_{2}\right) \geq 2 k+29$ except when $(n, k)$ is one of the following ten pairs:

$$
\begin{aligned}
& (91,3),(115,3),(115,4),(117,3),(143,3),(243,3),(341,3), \\
& (525,3),(663,3),(2871,3) .
\end{aligned}
$$


Proof. By Lemma 7, we need only consider $n$ such that

$M=n+4 j \quad$ for some $M \in T$ and some $j$ with $0 \leq j \leq(k-2) / 2$.

Then we find that

$$
2 k+29 \leq n \leq M \leq n+2(k-2) .
$$

Let $p$ be the largest prime $<M$ and $q$ the smallest prime $\geq \max \{2 k+29, M\}$. If $p \geq n$, then (2.3) implies that $p$ divides $\Delta_{2}$. If $p<n$ and $q \leq p+2 k$, then $q$ divides $\Delta_{2}$. Thus for any $k \geq(q-p) / 2$, the product $\Delta_{2}$ is divisible by either $p$ or $q$. So the assertion of the lemma is true provided $p \geq 2 k+29$. Thus we may assume that either

$$
k<\frac{q-p}{2} \quad \text { or } \quad k>\frac{p-29}{2} .
$$

Combining with $(2.3)$, we have

$$
\begin{aligned}
& \max \{2 k+29, M-2(k-2)\} \leq n \leq M, \\
& k<\min \left\{31, \frac{q-p}{2}\right\} \quad \text { or } \quad \frac{p-29}{2}<k \leq 30 .
\end{aligned}
$$

Thus for each $M \in T$, we check for the finitely many values of $(n, k)$ in (2.4) whether $P(\Delta) \geq 2 k+29$. We illustrate the above procedure with an example. Let $M=243$. Then $p=241$ and $q=251$. Hence

$$
k<5 \text { and } 239 \leq n \leq 243, \quad n \text { odd }
$$

In these cases we check directly that $P(\Delta) \geq 2 k+29$, the only exception being $(n, k)=(243,3)$. By the above procedure we find only the 10 exceptions listed in the statement of the lemma.

Finally we show

Lemma 9. Let $3 \leq k \leq 30$ and $n \geq 2 k+a$ with $1 \leq a<29$, a odd. Assume that $\Delta_{2}$ is not equal to any of the ten products in Lemma 8. Then

$$
P\left(\Delta_{2}\right)>2 k+a
$$

except when $(n, k, a) \in\{(23,3,17),(31,3,25)\}$.

Proof. By Lemmas 7 and 8 , we need to check the assertion only when

$$
2 k+a \leq n<2 k+29, \quad 1 \leq a<29, \quad a \text { odd }, \quad 3 \leq k \leq 30,
$$

which is done by direct computation.

For each odd $a \in[1,27]$, let $T(a)$ be the set of $M \in T$ for which $P(M(M+2)) \leq a+4 \leq M$. For example, when $a=1, T(a)=\{25\}$; when $a=3, T(a)=\{7,25,243\}$. Let $k$ be given and $n \geq 2 k+a$ with 
$1 \leq a<29, a$ odd. We denote by $a^{*}$ the smallest $a$ such that

$$
P\left(\Delta_{2}\right) \leq 2 k+a .
$$

With the above notation, we combine Lemmas 6 and 9 to obtain the following theorem.

TheOREM 8. Let $k \geq 2$ and $n \geq 2 k+a$ with $1 \leq a<29$, a odd. Then

$$
P\left(\Delta_{2}\right)>2 k+a
$$

except for the following values of $n, k$ and $a$ :

$$
\begin{aligned}
k=2,1 \leq a<29 \text { with } n \in T(a), \\
k=3,(n, a)=(23,17),(31,25), \\
k=3,\left(n, a^{*}\right)=(91,25),(115,17),(117,11),(143,23),(243,13), \\
\quad(341,25),(525,25),(663,23),(2871,23), \\
k=4,\left(n, a^{*}\right)=(115,15) .
\end{aligned}
$$

Proof. Let $k \geq 3$. Suppose $(n, k, a) \in\{(23,3,17),(31,3,25)\}$. Then $P\left(\Delta_{2}\right) \leq 2 k+a$ and these exceptions are listed in (2.6). Now assume that $(n, k, a) \notin\{(23,3,17),(31,3,25)\}$. Then by Lemmas 6 and 9 we find that $P\left(\Delta_{2}\right)>2 k+a$ except possibly when $(n, k)$ equals any of the ten pairs in Lemma 8. Let us take $(n, k)=(91,3)$. Then $P\left(\Delta_{2}\right)=31>2 k+a$ except when $a=25,27$. Thus $a^{*}=25$. Similarly $a^{*}$ for other pairs in Lemma 8 are found and listed in (2.6).

Now we take $k=2$. Then

$$
P(n(n+2)) \geq 37>2 k+a
$$

for all $n$ except those $n=M$ listed in $T$. For any given odd $a, 1 \leq a<29$, by our notation $T(a)$ denotes the values of $n \geq 2 k+a$ for which (2.5) does not hold. Hence $T(a)$ gives the set of exceptional values of $n$. This proves the theorem.

3. Newton polygons. As mentioned in the Introduction, a result of Dumas [3], from 1906, led Filaseta [6] to Lemma 1. Filaseta also remarks in [6] that this lemma may be strengthened by only requiring that $p$ not divide $a_{0} a_{m}$ in place of the condition that $\left|a_{0}\right|=\left|a_{m}\right|=1$; we make use of this stronger version of the lemma here. In [11, Shorey and Tijdeman gave a refinement of Lemma 1 using the notion of Newton function. Let $f$ be any polynomial of degree $n$ in $\mathbb{Z}[x]$. The Newton function $N f_{p}(x)$ with respect to a prime $p$ is a real valued function on the interval $[0, n]$ which has the Newton polygon of $f$ with respect to $p$ as its graph. We shall give below a slightly modified version of their lemma. 
Lemma 10. Let $k$ and $l$ be integers with $k>l \geq 0$. Suppose $u(x)=$ $\sum_{j=0}^{n} b_{j} x^{j} \in \mathbb{Z}[x]$ and $p$ is a prime such that $p \nmid b_{n}$ and $p \mid b_{j}$ for all $j \in$ $\{0,1, \ldots, n-l-1\}$. Let $a_{0}, a_{1}, \ldots, a_{n}$ be integers with $p \nmid a_{0} a_{n}$. Put $v(x)=$ $\sum_{j=0}^{n} a_{j} b_{j} x^{j}$. Then for any factor $h(x)$ of $v(x)$ having degree $k>l$, we have

$$
N u_{p}(y) \leq N h_{p}(y), \quad N u_{p}(n)-N u_{p}(n-k+y) \geq N h_{p}(k)-N h_{p}(y)
$$

for any $y \in[0, k]$ where $N h_{p}(k)$ is a positive integer.

Proof. Suppose

$$
v(x)=h(x) w(x)
$$

with deg $h(x)=k>l$. If the leftmost edge of the Newton polygon of $v(x)$ is of slope 0 , then its $x$-length is $\leq l$. Since $h(x)$ is of degree $k>l$, the Newton polygon of $h(x)$ has at least one edge of non-zero slope. Thus $N h_{p}(k)$ is a positive integer.

From the hypothesis it is clear that

$$
\left\{\begin{array}{l}
N u_{p}(0)=N v_{p}(0)=0, \quad N u_{p}(n)=N v_{p}(n), \\
N u_{p}(x) \leq N v_{p}(x) \quad \text { for } x \in(0, n)
\end{array}\right.
$$

Further, by Dumas' theorem, we have

$$
\left\{\begin{array}{l}
N v_{p}(y) \leq N h_{p}(y) \quad \text { for } y \in[0, k], \\
N v_{p}(n)-N v_{p}(n-k+y) \leq N u_{p}(n)-N u_{p}(n-k+y) \quad \text { for } y \in[0, k] .
\end{array}\right.
$$

Let us translate parallel to $x y$-axes the Newton polygon of $h$ defined on the interval $[0, k]$ so that the point $\left(k, N h_{p}(k)\right)$ coincides with $\left(n, N v_{p}(n)\right)$. Then the origin is shifted to $\left(n-k, N v_{p}(n)-N h_{p}(k)\right)$ and any $\left(x, N h_{p}(x)\right)$ for $x \in[0, k]$ goes to $\left(n-k+x, N h_{p}(x)+N v_{p}(n)-N h_{p}(k)\right)$. Thus the shifted Newton polygon of $h$ goes from $\left(n-k, N v_{p}(n)-N h_{p}(k)\right)$ to $\left(n, N v_{p}(n)\right)$ and it lies on or above the Newton polygon of $v$ in the interval $[n-k, n]$, by Dumas' theorem. Hence for any $x \in[0, k]$, we have

$$
N h_{p}(x)+N v_{p}(n)-N h_{p}(k) \geq N v_{p}(n-k+x)
$$

or

$$
N v_{p}(n)-N v_{p}(n-k+x) \geq N h_{p}(k)-N h_{p}(x) .
$$

Thus from (3.1) and (3.2) we get the assertion of the lemma.

Note that Lemma 1 follows from the above lemma, since, when the last edge of the Newton polygon of $g$ has slope $<1 / k$, then $N g_{p}(n)-N g_{p}(n-k)$ $<1$, by taking $u=g$ in the above lemma, from which we get $N h_{p}(k)=0$, a contradiction.

Lemma 11. Let $a, m$ and $k$ be positive integers with a odd and $k \leq m / 2$. Assume that there exists a prime $p>2 k+a$ dividing

$$
(a+2(m-k)) \cdots(a+2(m-1)) .
$$


Then the polynomial

$F(x)=x^{m}+(a+2(m-1)) x^{m-1}+\cdots+(a+2(m-1))(a+2(m-2)) \cdots a$

has no factor of degree $k$. Also the polynomial

$G(x)=a_{m} x^{m}+a_{m-1}(a+2(m-1)) x^{m-1}+\cdots+a_{0}(a+2(m-1))(a+2(m-2)) \cdots a$

has no factor of degree $k$, where $a_{0}, \ldots, a_{m}$ are integers with $p \nmid a_{0} a_{m}$.

Proof. Write $F(x)=x^{m}+c_{m-1} x^{m-1}+\cdots+c_{1} x+c_{0}$ and $G(x)=a_{m} x^{m}+$ $a_{m-1} c_{m-1} x^{m-1}+\cdots+a_{1} c_{1} x+a_{0} c_{0}$, where

$$
c_{j}=(a+2 j) \cdots(a+2(m-1)) \quad \text { for } 0 \leq j<m \quad \text { and } \quad c_{m}=1 .
$$

By assumption, there exists a prime $p>2 k+a$ dividing $c_{0}, c_{1}, \ldots, c_{m-k}$. From Lemma 1 we see that it suffices to show that the slope of the rightmost edge of the Newton polygon of $F(x)$ with respect to the prime $p$ is $<1 / k$. The slope of the rightmost edge is

$$
L=\max _{1 \leq j \leq m}\left\{\frac{\nu_{p}\left(c_{0}\right)-\nu_{p}\left(c_{j}\right)}{j}\right\} .
$$

Therefore $L$ equals

$$
\nu_{p}(a(a+2) \cdots(a+2(j-1))) / j \quad \text { for some } 1 \leq j \leq m .
$$

Let $j \leq k$. Since $p>2 k+a$, we see that

$$
\nu_{p}(a(a+2) \cdots(a+2(j-1))) / j=0<1 / k .
$$

Let $j>k$. Since $p \geq 2 k+a+2$, we have

$$
\nu_{p}(a(a+2) \cdots(a+2(j-1))) \leq \nu_{p}((a+2 j) !)<\frac{a+2 j}{p-1} \leq \frac{a+2 j}{a+1+2 k}<\frac{j}{k} .
$$

Thus, $L<1 / k$, as desired, completing the proof. and 11 .

Proof of Theorem 4. The assertion is a direct consequence of Lemmas 5

We combine Theorem 8 and Lemma 11 to obtain the following theorem.

Theorem 9. Let $a \geq 1$ be an odd integer less than 29, and let $m$ be any integer $\geq 2$. Assume that $2 m \neq n+2 k-a$ for any $(n, k, a)$ given by (2.6). Then the polynomial $F(x)$ in Lemma 11 has no factor of degree $\geq 2$. Further the polynomial $G(x)$ also has no factor of degree $\geq 2$ provided $a_{0}$ and $a_{m}$ are composed of primes $\leq a+4$.

4. Linear factors of $F(x)$ and $G(x)$. In this section we deal with the linear factors of $F(x)$ and $G(x)$. Again using Lemma 11, we show 
Lemma 12. Let $a \geq 1$ be an odd integer and let $m \geq 2$. Assume that there exists a prime $p$ such that

$$
p \nmid a, \quad p \mid(a+2(m-1)), \quad p^{1-1 /(p-1)} \geq(a+2)^{1 / 2} .
$$

Then the polynomial $F(x)$ has no linear factor. Also the polynomial $G(x)$ has no linear factor if such a prime $p$ does not divide $a_{0} a_{m}$.

Proof. Since $p$ divides $a+2(m-1)$, we see that $p \mid c_{j}$ for $0 \leq j<m$ where $c_{j}$ is given by (3.3). Further we require that $L<1$ where $L$ is given by (3.4). Thus we need

$$
\nu_{p}(a(a+2) \cdots(a+2(j-1)))<j \quad \text { for } 1 \leq j<m .
$$

Note that $\nu_{p}(a)=0$. Hence we may assume that $j>1$. Also we may suppose that $p \leq a+2(j-1)$, otherwise (4.1) holds since the left hand side of the inequality is 0 . Suppose $p^{\alpha} \leq a+2(j-1)<p^{\alpha+1}$. Then by taking blocks of $p, p^{2}, \ldots, p^{\alpha}$ successive terms we see that

$$
\begin{aligned}
\nu_{p}(a(a+2) \cdots(a+2(j-1))) & \leq\left(\left[\frac{j}{p}\right]+1\right)+\cdots+\left(\left[\frac{j}{p^{\alpha}}\right]+1\right) \\
& <\frac{j}{p-1}+\alpha \leq \frac{j}{p-1}+\frac{\log (a+2(j-1))}{\log p} .
\end{aligned}
$$

Thus 4.1) is valid if

$$
p^{j(1-1 /(p-1))} \geq a+2(j-1),
$$

which is true for $j \geq 2$ by the assumption on $p$. Now the result follows by Lemma 1.

5. Proof of Theorem 5. Suppose $g(x)$ has a factor of degree $k$ with $2 \leq k \leq m / 2$. By Theorem 9 we need only consider the exceptional values given in (2.6) with $n=M=a+2(m-k) \geq a+2 k$. To exclude these cases, we use Lemma 1. This requires finding a suitable prime so that

$$
p \mid c_{0}, \ldots, c_{m-k} \text { and } L<1 / k
$$

where $L$ is given by (3.4). For this, we use the following procedure.

Let $p$ be a prime such that

$$
p \mid c_{m-k}
$$

and $p \mid(a+2(m-\delta))$ with $\delta \geq 1$ chosen as small as possible.

Suppose $a+2 h$ is the least integer in $\{a, a+2, \ldots, a+2(m-1)\}$ divisible by $p$. Let $\nu_{p}(a+2(h+i p))=\theta_{i}$ for $0 \leq i \leq r=(m-\delta-h) / p$. Let

$$
L^{\prime}=\max \left\{\frac{\theta_{0}}{h+1}, \frac{\theta_{0}+\theta_{1}}{h+p+1}, \ldots, \frac{\theta_{0}+\cdots+\theta_{r}}{h+r p+1}\right\} .
$$


Then we see that

$$
\begin{aligned}
\nu_{p}\left(c_{0}\right) & =\cdots=\nu_{p}\left(c_{h}\right), \\
\nu_{p}\left(c_{h+1}\right) & =\cdots=\nu_{p}\left(c_{h+p}\right)=\nu_{p}\left(c_{0}\right)-\theta_{0}, \\
& \vdots \\
\nu_{p}\left(c_{h+(r-1) p+1}\right) & =\cdots=\nu_{p}\left(c_{h+r p}\right)=\nu_{p}\left(c_{0}\right)-\theta_{0}-\cdots-\theta_{r-1}, \\
\nu_{p}\left(c_{h+r p+1}\right) & =\cdots=\nu_{p}\left(c_{m-1}\right)=\nu_{p}\left(c_{0}\right)-\theta_{0}-\cdots-\theta_{r} .
\end{aligned}
$$

Since $\theta_{r}>0$, we have $L^{\prime}>0$. Thus by definition, $L=L^{\prime}$.

Assume that

$$
\frac{\theta_{0}}{h+1}<\frac{1}{k} \quad \text { and } \quad \theta_{i}<\frac{p}{k} \quad \text { for } 1 \leq i \leq r .
$$

Then

$$
\begin{aligned}
\frac{\theta_{0}+\theta_{1}}{h+p+1} & =\frac{\theta_{0}}{h+1} \frac{h+1}{h+p+1}+\frac{\theta_{1}}{h+p+1} \\
& <\frac{h+1}{k(h+p+1)}+\frac{p}{k(h+p+1)}=\frac{1}{k}
\end{aligned}
$$

and by induction, we see that

$$
\frac{\theta_{0}+\cdots+\theta_{s}}{h+s p+1}<\frac{1}{k} \quad \text { for } 1<s \leq r .
$$

Thus $L<1 / k$, which is required in (5.1). Thus we need only satisfy (5.3). Since

$$
p^{\theta_{i}} \leq a+2(h+i p) \leq a+2(m-1)=M+2(k-1),
$$

condition $(5.3)$ is satisfied if

$$
\frac{\theta_{0}}{h+1}<\frac{1}{k} \quad \text { and } \quad M+2(k-1)<p^{p / k} .
$$

Thus we need only choose a prime $p$ satisfying 5.2 and (5.4). In Tables 2 and 3 , we give a choice of $p$ for most of the values of $(M ; a)$ listed in $(2.6)$. Note that the choice of $p$ is not unique. When $k=4$, we have $(M ; a)=$ $(115 ; 15-27)$. We exclude this case by taking $p=13$ so that $a+2 h=39$ giving $6 \leq h \leq 12$ and $\theta_{0}=1$, which satisfy (5.2) and (5.4).

Table $2(k=3)$

\begin{aligned} & \hline \multicolumn{1}{c}{$(M ; a)$} \\ & \hline 7$(31 ; 25) \\ & 11(117 ; 13),(143 ; 23-27),(341 ; 25-27),(2871 ; 23-27) \\ & 13(91 ; 25-27),(115 ; 17-27),(117 ; 15-27),(243 ; 15-27),(663 ; 23-27) \\ & 17(117 ; 11),(525 ; 25-27) \\ & 19(243 ; 13) \\ & 23(23 ; 17)\end{aligned}$


Table $3(k=2)$

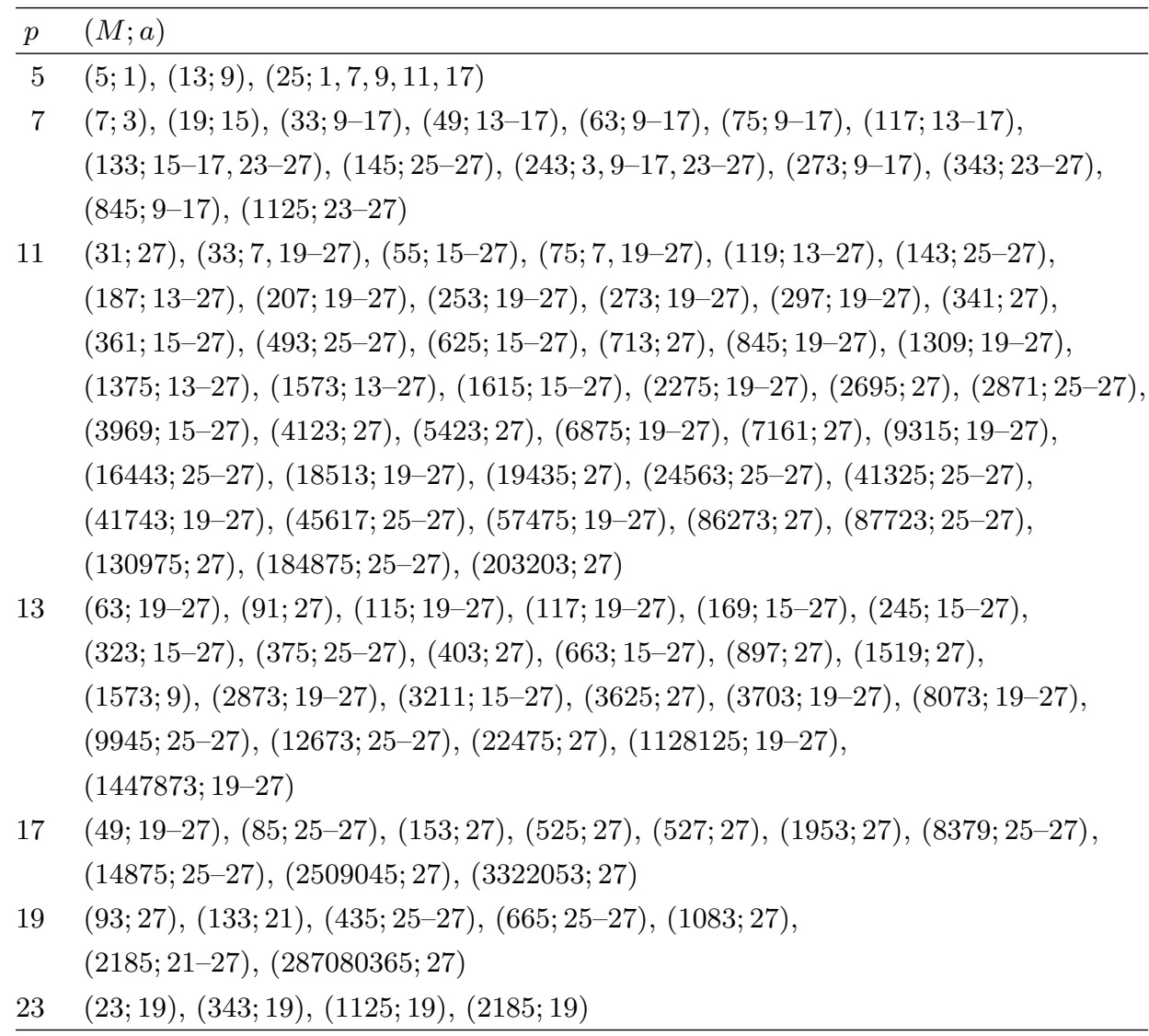

For the choices of $p$ given in Tables 2 and 3, conditions (5.2) and (5.4) are satisfied and thus all these values are excluded. When $k=2$ and $(M ; a)=$ $(243 ; 7),(1573 ; 11)$, we take $p=5,7$, respectively, and compute $L^{\prime}$ to get $L<1 / k$. Hence these cases are also excluded. Thus all values of $(M ; a)$ given in (2.6) are excluded except when

$$
\begin{aligned}
(M ; a) \in\{ & (25 ; 3,5,13,15,19,21),(133 ; 19),(243 ; 5,19,21), \\
& (343 ; 21),(1125 ; 21)\} .
\end{aligned}
$$

Next we illustrate the application of Lemma 10 with an example. Let $(M ; a)=(25 ; 3)$. Then $m=13$ and $u(x)=x^{13}+27 x^{12}+\cdots+3 \cdot 5 \cdots 27$. The vertices of the Newton polygon of $u(x)$ with respect to the prime $p=3$ are

$$
(0,0)-(9,5)-(12,7)-(13,8)
$$

By Lemma 10, any quadratic factor $h(x)$ satisfies $N h_{3}(2) \geq N u_{3}(2)=10 / 9$ 
implying $N h_{3}(2) \geq 2$. Also we have

$$
N u_{3}(13)-N u_{3}(11)=8-19 / 3=5 / 3 \geq N h_{3}(2),
$$

which is a contradiction. Thus the case $(M ; a)=(25 ; 3)$ is excluded. Below we give the values of $(M ; a, p)$ together with the vertices of the corresponding Newton polygon which are excluded by Lemma 10.

$$
\begin{array}{ll}
(25 ; 5,3): & (0,0)-(9,5)-(12,7), \\
(25 ; 13,3): & (0,0)-(8,5), \\
(25 ; 15,3): & (0,0)-(6,4)-(7,5), \\
(25 ; 19,3): & (0,0)-(5,4) .
\end{array}
$$

As already noted, in the case $(M ; a)=(25 ; 21)$ there are reducible polynomials. Thus we are left with six undecided cases in (5.5).

In these cases including $(M ; a)=(25 ; 21)$ we check directly with MaTHEMATICA that the resulting polynomials $f(x)$ do not factor. Thus $f(x)$ has no factors of degree $\geq 2$. This completes the proof of the theorem.

Acknowledgments. The authors would like to thank Professor Michael Filaseta for many helpful discussions. The second author also wishes to thank him for his kind hospitality during her visit to the University of South Carolina in May-June, 2007. We thank Professors T. N. Shorey and R. Tijdeman for providing us with the preprint of their paper [11. We also owe our sincere thanks to the referee for his/her helpful comments and for pointing out a rectifiable error in the earlier version of the paper.

\section{References}

[1] M. Allen and M. Filaseta, A generalization of a second irreducibility theorem of I. Schur, Acta Arith. 109 (2003), 65-79.

[2] —, -, A generalization of a third irreducibility theorem of I. Schur, ibid. 114 (2004), 183-197.

[3] G. Dumas, Sur quelques cas d'irréductibilité des polynômes à coefficients rationnels, J. Math. Pures Appl. 2 (1906), 191-258.

[4] P. Dusart, Autour de la fonction qui compte le nombre de nombres premiers, Ph.D. thesis, Université de Limoges, 1998.

[5] E. F. Ecklund, Jr., R. B. Eggleton, P. Erdős and J. L. Selfridge, On the prime factorization of binomial coefficients, J. Austral. Math. Soc. Ser. A 26 (1978), 257-269.

[6] M. Filaseta, The irreducibility of all but finitely many Bessel polynomials, Acta Math. 174 (1995), 383-397.

[7] - A generalization of an irreducibility theorem of I. Schur, in: Analytic Number Theory, Proc. Internat. Conf. in Honor of Heini Halberstam, Vol. 1, B. C. Berndt, H. G. Diamond and A. J. Hildebrand (eds.), Birkhäuser, Boston, 1996, 371-395.

[8] D. H. Lehmer, On a problem of Störmer, Illinois J. Math. 8 (1964), 57-79. 
[9] N. Saradha and T. N. Shorey, Almost perfect powers in arithmetic progression, Acta Arith. 99 (2001), 363-388.

[10] I. Schur, Einige Sätze über Primzahlen mit Anwendungen auf Irreduzibilitätsfragen, I, II, Sitzungsber. Preuss. Akad. Wiss. Berlin Phys.-Math. Kl. 1929, 125-136, 370-391.

[11] T. N. Shorey and R. Tijdeman, Generalizations of some irreducibility results by Schur, preprint.

Carrie E. Finch

Mathematics Department

Washington and Lee University

Lexington, VA 24450, U.S.A.

E-mail: finchc@wlu.edu
N. Saradha

School of Mathematics

Tata Institute of Fundamental Research

Homi Bhabha Road

Mumbai, 400 005, India

E-mail: saradha@math.tifr.res.in

Received on 24.5.2008

and in revised form on 13.1.2010 